\title{
Phenomenology of chiral damping in noncentrosymmetric magnets
}

\author{
Collins Ashu Akosa, ${ }_{1}^{1}$ Ioan Mihai Miron, ${ }^{2,3,4}$ Gilles Gaudin, ${ }^{2,3,4}$ and Aurélien Manchon ${ }^{1, *}$ \\ ${ }^{1}$ King Abdullah University of Science and Technology (KAUST), \\ Physical Science and Engineering Division (PSE), Thuwal 23955, Saudi Arabia \\ ${ }^{2}$ Université Grenoble Alpes, INAC-SPINTEC, F-38000 Grenoble, France \\ ${ }^{3}$ CNRS INAC-SPINTEC, F-38000 Grenoble, France \\ ${ }^{4}$ CEA INAC-SPINTEC, F-38000 Grenoble, France \\ (Received 28 July 2015; published 21 June 2016)
}

\begin{abstract}
A phenomenology of magnetic chiral damping is proposed in the context of magnetic materials lacking inversion symmetry. We show that the magnetic damping tensor acquires a component linear in magnetization gradient in the form of Lifshitz invariants. We propose different microscopic mechanisms that can produce such a damping in ferromagnetic metals, among which local spin pumping in the presence of an anomalous Hall effect and an effective " $s$ - $d$ " Dzyaloshinskii-Moriya antisymmetric exchange. The implication of this chiral damping in terms of domain-wall motion is investigated in the flow and creep regimes.
\end{abstract}

DOI: 10.1103/PhysRevB.93.214429

\section{INTRODUCTION}

Understanding energy relaxation processes of fast dissipative systems at the nanoscale is of paramount importance for the smart design and operation of ultrafast nanodevices. In this respect, magnetic heterostructures have drawn increasing enthusiasm in the past ten years with the subpicosecond optical control of magnetic order [1,2] and the promises of ultrafast domain-wall motion in asymmetric metallic multilayers [3]. In fact, the observation of ultrahigh current-driven velocity in ultrathin multilayers has come as a surprise and triggered intense investigations on the physics emerging from symmetry breaking in strong spin-orbit coupled magnets [4,5]. These studies have unravelled the essential role played by Dzyaloshinskii-Moriya interaction [6,7] (DMI), an antisymmetric exchange interaction that emerges in magnets lacking spatial inversion symmetry. This interaction forces neighboring spin to align perpendicular to each other and competes with the ferromagnetic exchange, resulting in distorted textures such as spin spirals or Skyrmions, as observed in bulk inversion asymmetric magnets [8] as well as at the interface of transition metals $[9,10]$. In perpendicularly magnetized domain walls, this interaction favors a Néel over Bloch configuration [11-13], a key ingredient explaining most of the thought-provoking observations reported to date [14]. The dynamics of spin waves can also be modified by DMI, which distorts the energy dispersion [15] and results in a relaxation that depends on the propagation direction $[16,17]$.

A crucial aspect of fast dynamical processes is the nature of the energy relaxation. In the hydrodynamic limit of magnetic systems, this dissipation is written in the form of a nonlocal tensor [see Eq. (1)] whose complex physics is associated with a wide variety of mechanisms such as many-magnon scattering [18] and itinerant electron spin relaxation [19]. Since the energy relaxation rate of spin waves depends on their wave vector (the higher the spin wave energy, the stronger its dissipation), the magnetic damping of smooth magnetic textures (i.e., in the long wavelength limit $q$ ) depends on

\footnotetext{
*aurelien.manchon@kaust.edu.sa
}

the inverse of the exchange length, $q \sim 1 / \lambda_{\mathrm{dw}}$. In inversion symmetric systems, this results in a correction to the magnetic damping of the order of $1 / \lambda_{\mathrm{dw}}^{2}$ [20-22]. However, in magnetic systems lacking inversion symmetry, such as the systems in which DMI is observed, the energy dissipation becomes chiral: a component linear in the magnetization gradient emerges, thereby fulfilling Neumann's principle stating that "any physical properties of a system possess the symmetry of that system."

In the present work, we explore the nature of the magnetic damping in noncentrosymmetric magnets and propose different physical mechanisms resulting in the emergence of a chiral damping. This chiral damping significantly impacts the motion of magnetic domain walls in both flow and creep regimes, opening appealing avenues to solve recent puzzling observations [23].

\section{SYMMETRY CONSIDERATIONS}

The equation of motion governing the dynamics of continuous magnetic textures is given by the Landau-Lifshitz-Gilbert (LLG) equation,

$$
\partial_{t} \mathbf{m}=-\gamma \mathbf{m} \times \mathbf{H}_{\text {eff }}+\mathbf{m}(\mathbf{r}) \times \int d \mathbf{r}^{\prime} \underline{\underline{\alpha}}\left(\mathbf{r}, \mathbf{r}^{\prime}\right) \partial_{t} \mathbf{m}\left(\mathbf{r}^{\prime}\right),
$$

where $\mathbf{m}(\mathbf{r}, t)=\mathbf{M}(\mathbf{r}, t) / M_{s}$ is a unit vector in the direction of the magnetization $\mathbf{M}(\mathbf{r}, t), \gamma$ is the gyromagnetic ratio, $\mathbf{H}_{\text {eff }}$ is the effective field incorporating the external applied, anisotropy, exchange, DMI, and demagnetizing fields, and $\underline{\boldsymbol{\alpha}}\left(\mathbf{r}, \mathbf{r}^{\prime}\right)$ is the magnetic damping expressed as a nonlocal second-rank tensor. In the limit of smooth textures, the tensorial components of the damping is a function of the magnetization direction and of its spatial gradients, $\alpha^{i j}=$ $\alpha^{i j}(\mathbf{m}, \nabla \mathbf{m})$. Performing an expansion up to the first order in the magnetization gradient yields [24]

$$
\alpha^{i j}=\alpha_{0}^{i j}+\sum_{l m} K_{l m}^{i j} m_{l} m_{m}+\sum_{k l m} L_{k l m}^{i j} m_{k} \partial_{l} m_{m} .
$$

The first term is the isotropic damping, the second term amounts for the magnetocrystalline anisotropy, and the third term is the chiral damping. Only terms bilinear in 
magnetization direction $m_{i}$, i.e., even under time-reversal symmetry, are retained in the expansion. Spatial inversion symmetry breaking imposes the third term of Eq. (2) to reduce to a sum of Lifshitz antisymmetric invariants, $\propto m_{k} \partial_{l} m_{m}-$ $m_{m} \partial_{l} m_{k}$ (i.e., $L_{k l m}^{i j}=-L_{m l k}^{i j}$ ). In the case of a cubic threedimensional system with bulk spatial inversion symmetry breaking (e.g., B20 or ZnS crystals), all three crystallographic directions $(\mathbf{x}, \mathbf{y}$, and $\mathbf{z})$ are equivalent and the chiral damping adopts the general form

$$
\alpha^{i j}=\alpha_{0}^{i j}+\alpha_{3 d}^{i j} \lambda_{\mathrm{dw}} \mathbf{m} \cdot(\nabla \times \mathbf{m}),
$$

where $\lambda_{\mathrm{dw}}$ defines the characteristic exchange length. In a twodimensional system with interfacial symmetry breaking along $\mathbf{z}$, i.e., invariant under $\mathcal{C}_{\infty z}$ rotation symmetry, the damping takes the form

$$
\alpha^{i j}=\alpha_{0}^{i j}+\alpha_{z}^{i j} \lambda_{\mathrm{dw}} \mathbf{m} \cdot\left(\nabla_{\mathbf{z}} \times \mathbf{m}\right),
$$

where $\nabla_{\mathbf{z}}=\mathbf{z} \times \nabla$.

As dictated by Neumann's principle, the chiral damping possesses the same symmetry as DMI for these systems (e.g., see Refs. [8] and [14]). In addition, Onsager reciprocity imposes $\alpha^{i j}=\alpha^{j i}$, and hence, one can construct a chiral damping up to linear order in the magnetization gradient based on symmetry arguments. These considerations suggest that such a damping is present in noncentrosymmetric (ferro-, ferri-, and antiferro-)magnets in general, not limited to metals. However, this phenomenology does not provide information regarding the strength of the chiral damping itself, the relative values of the off-diagonal tensor elements $\left(\sim \alpha^{i \neq j}\right)$ compared to the diagonal ones $\left(\sim \alpha^{i i}\right)$, nor does it indicate the underlying physical mechanisms responsible for it. Let us now turn our attention towards the microscopic origin of such chiral damping.

\section{MICROSCOPIC ORIGIN OF THE CHIRAL DAMPING}

We focus our attention on magnetic metals where the damping is driven by the spin relaxation of itinerant electrons [19]. In ferromagnets with interfacial Rashba spin-orbit coupling, it has been shown that the magnetic damping adopts the form of a tensor, linear in both magnetization gradient and Rashba strength [25-27]. Besides this effect, we here propose two additional mechanisms that can contribute to the chiral damping.

The first mechanism is illustrated in Fig. 1(a) and arises from the interplay between spin motive force and anomalous Hall effect. Indeed, it has been shown recently that timedependent spin textures generate a local spin current $\mathbf{J}_{i}^{s}=$ $\frac{g \mu_{B} \hbar G_{0}}{4 e^{2}} \partial_{t} \mathbf{m} \times \partial_{i} \mathbf{m}$ [28], where $G_{0}$ is the electrical conductivity. This spin current flows along $\mathbf{e}_{i}$ direction and is spin polarized along $\partial_{t} \mathbf{m} \times \partial_{i} \mathbf{m} \propto \mathbf{m}$. It induces magnetic dissipation at the second order of the spatial gradient [20]. Now, when anomalous Hall effect is present in the ferromagnet, a charge current $\mathbf{J}_{e}$ can be converted into a charge Hall current of the form $\mathbf{J}_{\mathrm{H}}=\theta_{\mathrm{H}} P \mathbf{m} \times \mathbf{J}_{e}$, where $\theta_{\mathrm{H}}$ is the spin Hall angle and $P$ is the spin polarization in the ferromagnet. Following the same mechanism, the primary spin current $\mathbf{J}_{i}^{S}$ can be converted into a secondary spin current with the same polarization $\sim \mathbf{m}$ but that flows along $\mathbf{m} \times \mathbf{e}_{i}\left(\equiv \mathbf{J}_{i}^{s} \times \mathbf{e}_{i}\right)$. This secondary spin
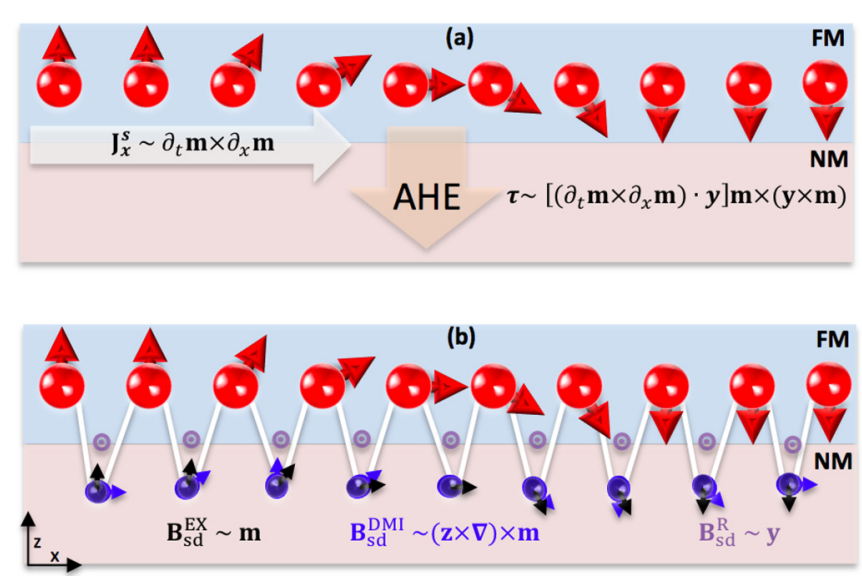

FIG. 1. Illustration of different microscopic mechanisms at the origin of chiral damping. In (a), the spin current induced by the moving magnetization is dissipated in the adjacent metal via anomalous Hall effect. Panel (b) illustrates the chiral damping process due to $s$ - $d$ DMI. The itinerant electron spins (black arrows) feel three competing effective fields: the isotropic exchange field $\mathbf{B}_{\mathrm{sd}}^{\mathrm{ex}} \sim \mathbf{m}$ in the direction of the local moments (red), the constant Rashba field $\mathbf{B}_{\text {sd }}^{\mathrm{R}} \sim \mathbf{y}$ (purple), and the spatially inhomogeneous DM field $\mathbf{B}_{\text {sd }}^{\mathrm{DMI}} \sim(\mathbf{z} \times \nabla) \times \mathbf{m}$ (blue). This field, which varies in both magnitude and direction, results in a damped precession that depends on the chirality of the wall.

current reads $\mathbf{J}_{j}^{s}=\theta_{\mathrm{H}} P\left[\mathbf{J}_{i}^{s} \cdot\left(\mathbf{e}_{i} \times \mathbf{e}_{j}\right)\right] \mathbf{e}_{i} \times \mathbf{e}_{j}$. The secondary spin current, which flows along $\mathbf{e}_{j}$ and is spin polarized along $\mathbf{e}_{i} \times \mathbf{e}_{j}$, can be injected into an adjacent spin sink with strong spin relaxation, thereby inducing a dissipative torque on the magnetization. The latter is very similar to the well-known spin pumping mechanism [29]. Considering a one-dimensional domain wall along $\mathbf{x}$ deposited on a heavy metal with an interface normal to $\mathbf{z}$, we obtain a damping torque of the form [30]

$$
\boldsymbol{\tau}=\mathcal{A} \frac{\theta_{\mathrm{H}} g \mu_{B} \hbar P G_{0}}{4 e^{2} M_{s} d}\left[\left(\partial_{t} \mathbf{m} \times \partial_{i} \mathbf{m}\right) \cdot \mathbf{y}\right] \mathbf{m} \times(\mathbf{y} \times \mathbf{m}),
$$

$\mathcal{A}$ being a renormalization factor arising from the spin current backflow ( $\mathcal{A} \rightarrow 1$ for strong spin relaxation [29]), $M_{s}$ the saturation magnetization, and $d$ the thickness of the ferromagnet. Assuming $G_{0}=2 \times 10^{7} \mathrm{~S} \mathrm{~m}^{-1}, P=0.7, \theta_{\mathrm{H}}=5 \%$, $d=1 \mathrm{~nm}$, and $M_{s}=800 \mathrm{emu} / \mathrm{cc}$, we find a chiral damping of the order of 0.015 for a domain-wall thickness $\lambda_{\mathrm{dw}}=1 \mathrm{~nm}$. This damping torque is proportional to $\sim \sin 2 \varphi, \varphi$ being the azimuthal angle of the magnetization, and vanishes when the wall is either in Bloch $(\varphi=\pi / 2)$ or Néel configuration $(\varphi=0)$.

The second mechanism is related to DMI. As demonstrated in Ref. [31], in transition metal ferromagnets both localized ( $p d$ hybridized) and delocalized electrons ( $s p d$ hybridized) contribute to DMI and therefore one can parse the total spin moment $\mathbf{S}_{i}$ into localized ( $d$-dominated) and delocalized ( $s$-dominated) contributions $\mathbf{S}_{i}=\mathbf{S}_{i}^{d}+\hat{\mathbf{S}}_{i}^{s}$. The resulting DMI between sites $i$ and $j$ can be phenomenologically rewritten up to the linear order in $\hat{\mathbf{S}}^{s}$ as $\mathbf{D}_{i j} \cdot \mathbf{S}_{i} \times \mathbf{S}_{j}=\mathbf{D}_{i j}^{d d} \cdot \mathbf{S}_{i}^{d} \times \mathbf{S}_{j}^{d}+$ $\mathbf{D}_{i j}^{s d} \cdot \mathbf{S}_{i}^{d} \times \hat{\mathbf{S}}_{j}^{s}$ (neglecting a higher-order term $\sim \hat{\mathbf{S}}_{i}^{s} \times \hat{\mathbf{S}}_{j}^{s}$ ). The first term involves orbital overlap between localized states, while the second term describes the chiral exchange between 
the local and itinerant spins. In the continuous limit, the Hamiltonian of the itinerant electrons can be written as

$$
\hat{\mathcal{H}}_{\mathrm{sd}}=\frac{\hat{\mathbf{p}}^{2}}{2 m}+J_{\mathrm{ex}} \mathbf{m} \cdot \hat{\boldsymbol{\sigma}}+\frac{\alpha_{\mathrm{R}}}{\hbar} \hat{\mathbf{p}}_{\mathbf{z}} \cdot \hat{\boldsymbol{\sigma}}+\frac{\alpha_{\mathrm{D}}}{\hbar}\left(\hat{\mathbf{p}}_{\mathbf{z}} \times \mathbf{m}\right) \cdot \hat{\boldsymbol{\sigma}}
$$

where $\hat{\mathbf{p}}_{\mathbf{z}}=\mathbf{z} \times \hat{\mathbf{p}}, \hat{\boldsymbol{\sigma}}$ is the vector of Pauli spin matrices, $J_{\mathrm{ex}}$, $\alpha_{\mathrm{R}}$, and $\alpha_{\mathrm{D}}=D_{\mathrm{sd}} a$ ( $a$ is the lattice parameter) are the strength of the exchange, Rashba spin-orbit, and $s-d$ DM interactions, respectively. Equation (6) suggests that itinerant spins flowing through the magnetic texture illustrated in Fig. 1(b) experience three types of effective magnetic fields (see also Ref. [32]): the inhomogeneous exchange field $\sim \mathbf{m}$, the constant Rashba field $\sim \mathbf{y}$, and the inhomogeneous, texture-dependent $s$ - $d \mathrm{DM}$ field $\sim(\mathbf{z} \times \nabla) \times \mathbf{m}$. Due to the cooperation of the second and third terms in Eq. (6), the Fermi surface becomes dependent on the magnetization direction, a phenomenon known as Fermi surface breathing [19] and responsible for conventional (possibly anisotropic) magnetic damping. The last term, $s-d$ DMI, introduces a breathing that depends on the magnetization gradient and therefore promotes chiral damping.

To derive the equation of motion of the itinerant electron spin density $\mathbf{s}=\langle\hat{\boldsymbol{\sigma}}\rangle$ from Eq. (6), we follow a standard procedure [30] and obtain

$$
\partial_{t} \mathbf{s}+\nabla \cdot \mathcal{J}+\frac{1}{\tau_{\mathrm{ex}}} \mathbf{S} \times \mathbf{m}=\nabla_{\mathbf{z}}\left[b_{\mathrm{R}}+\times b_{\mathrm{D}} \mathbf{m}\right] \times \mathbf{s}-\Gamma_{\mathrm{re}},
$$

where $\tau_{\mathrm{ex}}=\hbar / 2 J_{\mathrm{sd}}$ is the spin precession time, $b_{\mathrm{R}}=2 \alpha_{\mathrm{R}} / \hbar$, and $b_{\mathrm{D}}=2 \alpha_{\mathrm{D}} / \hbar . \mathcal{J}=-\mathcal{D} \nabla \otimes \mathbf{s}$ is the spin current density tensor, $\mathcal{D}$ being the diffusion constant. The solution of Eq. (7) for the spin density and torque is nontrivial. Therefore, we proceed with the gradient expansion method within the relaxation time approximation (see Ref. [30] for details) to obtain the torque $\tau$ generated by a precessing magnetization $\sim \partial_{t} \mathbf{m}$ :

$$
\begin{aligned}
\boldsymbol{\tau} / \tilde{n}_{s} \approx & (1+\xi \chi-\beta \mathbf{m} \times)\left[-\partial_{t} \mathbf{m}\right. \\
& +\lambda_{\mathrm{R}}\left[\nabla_{\mathbf{z}} \times\left(\mathbf{m} \times \partial_{t} \mathbf{m}+\xi \partial_{t} \mathbf{m}\right)\right]_{\perp} \\
& \left.+\lambda_{\mathrm{D}}\left[\left(\nabla_{\mathbf{z}} \times \mathbf{m}\right) \times\left(\mathbf{m} \times \partial_{t} \mathbf{m}+\xi \partial_{t} \mathbf{m}\right)\right]_{\perp}\right] .
\end{aligned}
$$

In this expression, $\beta=\tau_{\mathrm{ex}} / \tau_{\mathrm{sf}}, \chi=\tau_{\mathrm{ex}} / \tau_{\varphi}, \xi=\chi+\beta, \tilde{n}_{s}=$ $n_{s} /\left(1+\xi^{2}\right)$, and the subscript $\perp$ indicates that the torque is defined perpendicular to the magnetization $\mathbf{m}$. The first term has been derived previously [33], the second term $\left(\lambda_{\mathrm{R}}=\tau_{\mathrm{ex}} b_{\mathrm{R}}\right)$ arises from Rashba spin-orbit coupling [25,26], and the third term $\left(\lambda_{\mathrm{D}}=\tau_{\mathrm{ex}} b_{\mathrm{D}}\right)$ arises from the $s-d$ DMI exchange. The total torque $\tau$ contributes both to the renormalization of the gyromatic ratio (terms that preserve time-reversal symmetry) and to dissipation (terms that break time-reversal symmetry). This form is more general than Eq. (2). In fact, the expansion given in Eq. (2) assumes a damping torque of the form $\boldsymbol{\tau} \sim$ $\mathbf{m} \times \underline{\alpha} \cdot \partial_{t} \mathbf{m}$, where $\underline{\alpha}$ is a space-dependent tensor. In contrast, Eq. $(\overline{\overline{8}})$ is the explicit form of the damping torque calculated microscopically and involving terms, such as $\sim \nabla \partial_{t} \mathbf{m}$, that are absent from Eq. (2).

Assuming $\alpha_{\mathrm{R}} \approx 10^{-11} \mathrm{eV} \mathrm{m} \mathrm{[3],} \alpha_{\mathrm{D}} \approx D_{\mathrm{sd}} a=10^{-11} \mathrm{eV} \mathrm{m}$ [31], and $\tau_{\mathrm{ex}} \approx 10^{-14} \mathrm{~s}$, we obtain $\lambda_{\mathrm{R}}=\lambda_{\mathrm{D}}=0.3 \mathrm{~nm}$. Considering that $\tilde{n}_{s}=0.1$ and $\tau_{\varphi} \approx \tau_{\mathrm{sf}} \approx 10^{-13} \mathrm{~s}$, the chiral damping is of the order of $\tilde{n}_{s} \lambda_{\mathrm{R}} / \lambda_{\mathrm{dw}} \approx 0.03$ and $\tilde{n}_{s} \xi \lambda_{\mathrm{D}} / \lambda_{\mathrm{dw}} \approx 0.003$ for $\lambda_{\mathrm{dw}}=1 \mathrm{~nm}$. Notice that the chiral damping arising from $s$ - $d$ DMI $\left(\propto D_{\text {sd }}\right)$ does not necessarily scale with the DMI itself $\left(\propto D_{\mathrm{dd}}\right)$, as both effects, chiral damping and asymmetric exchange, involve different electron orbitals.

\section{DOMAIN-WALL MOTION}

To illustrate the effect of this chiral damping on the dynamics of magnetic textures, we first derive the equation of motion of a one-dimensional perpendicular domain wall, commonly observed in heavy metal/ferromagnet asymmetric multilayers [3-5]. The magnetization is defined $\mathbf{m}=(\cos \varphi \sin \theta, \sin \varphi \sin \theta, \cos \theta)$, where $\varphi=\varphi(t)$ is the azimuthal angle and $\theta(x)=2 \tan ^{-1}\left(\exp \left[s(x-X) / \lambda_{\mathrm{dw}}\right]\right), X$ being the domain-wall center, and $s= \pm 1$ defining the domain-wall chirality ( $\uparrow \downarrow$ or $\downarrow \uparrow$, respectively). The magnetic domain wall is submitted to a magnetic anisotropy field $\mathbf{H}_{k}=H_{k} \sin \theta \sin \varphi \mathbf{y}$ which favors a Bloch configuration and an applied magnetic field $\mathbf{H}=H_{x} \mathbf{x}$ which favors a Néel configuration. The damping torque is given by Eq. (8), and the rigid domain-wall dynamics is described by the coupled equations

$$
\begin{aligned}
\partial_{\tau} \varphi & =H_{\mathrm{z}}+s \frac{\pi}{2}(\alpha-s \mu \cos \varphi)\left(H_{x} \sin \varphi-\frac{H_{k}}{2} \sin 2 \varphi\right), \\
\frac{s \partial_{\tau} X}{\lambda_{\mathrm{dw}}} & =(\alpha-s \nu \cos \varphi) H_{\mathrm{z}}-\frac{\pi}{2}\left(H_{x} \sin \varphi-\frac{H_{k}}{2} \sin 2 \varphi\right),
\end{aligned}
$$

where we defined $\tau=\gamma t, \mu=\left(\lambda_{\mathrm{D}}-\xi \lambda_{\mathrm{S}}\right)\left(\pi \tilde{n}_{s} / 4 \lambda_{\mathrm{dw}}\right)$, and $v=\left(\beta \lambda_{\mathrm{D}}+\lambda_{\mathrm{S}}\right)\left(\pi \tilde{n}_{\mathrm{s}} / 4 \lambda_{\mathrm{dw}}\right)(\alpha, \nu, \mu \ll 1)$. In Eq. (9), we neglected renormalization of the gyromagnetic ratio and only considered the dissipative components. The damping due to $s$ - $d$ DMI and Rashba spin-orbit coupling both produce a contribution $\propto s \cos \varphi$ that depends on the domain-wall chirality $s$ and on the azimuthal angle $\varphi$. Notice that the DM field does not explicitly enter these equations since it can be simply modeled by a chiral in-plane field $\sim_{s} H_{x}$ [14].

Let us now investigate the influence of this damping on the field-driven motion of a domain wall submitted to both perpendicular $\left(H_{z}\right)$ and in-plane $\left(H_{x}\right)$ magnetic fields. In this example, we chose $\mu=v=\alpha_{\mathrm{c}}$ for simplicity. Figure 2(a) shows the steady-state velocity of the domain wall as a function of $H_{z}$ for different chiral damping strengths and $H_{x}=0$. These velocity curves display the usual Walker breakdown around $H_{z} \approx 12-19 \mathrm{mT}$. Below Walker breakdown and in the absence of in-plane field $H_{x}$, the domain-wall azimuthal angle $\varphi$ obeys $H_{z}=s \frac{\pi}{4} H_{k}\left(\alpha-s \alpha_{c} \cos \varphi\right) \sin 2 \varphi$, which produces a kink around $H_{z} \approx s \alpha_{c} H_{\mathrm{WB}} / \alpha$ where $H_{\mathrm{WB}}=\alpha \pi H_{k} / 4$, which is associated with a jump in the effective damping, $\alpha_{\text {eff }}=\alpha-$ $\alpha_{c} \cos \varphi$ [see Fig. 2(c)]. When the in-plane field $H_{x}$ is applied and exceeds the anisotropy field $H_{k}$, a Néel configuration is stabilized $(\varphi \approx 0, \pi)$ and the damping becomes constant $\alpha_{\text {eff }}=$ $\alpha \pm \alpha_{c}$. Hence, the kink disappears as shown in Fig. 2(b). The dependence of the velocity as a function of $H_{x}$ at fixed $H_{z}$ is even more revealing. By sweeping $H_{x}$, the domain wall is tuned from a Bloch to Néel configuration by the in-plane field [see Figs. 2(d)-2(f)]. Hence, the effective damping is strongly modified [Fig. 2(f)] and the domain-wall velocity becomes asymmetric, as reported in Fig. 2(d). Notice that the kink is still observable at a small negative in-plane field. 

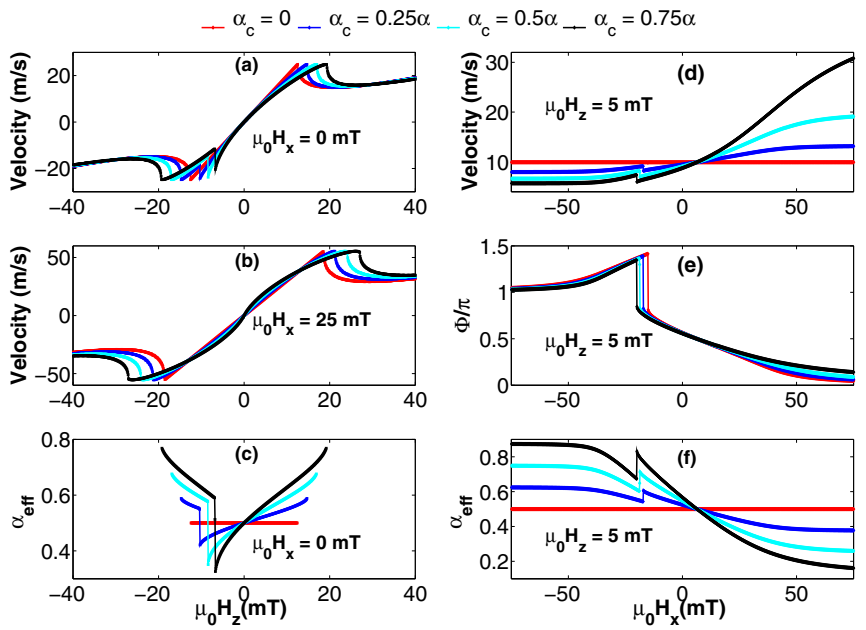

FIG. 2. (a,b) Domain-wall velocity as a function of perpendicular magnetic field for different chiral damping strengths at $H_{x}=0 \mathrm{mT}$ and $H_{x}=25 \mathrm{mT}$, respectively; (c) corresponding effective damping. (d) Domain-wall velocity as a function of in-plane magnetic field for different chiral damping strengths, and for $H_{z}=5 \mathrm{mT}$; (e,f) corresponding azimuthal angle and effective damping, respectively. The parameters are $\alpha=0.5, H_{\mathrm{WB}}=12.5 \mathrm{mT}$. In all simulations, we start with a Bloch configuration $(\varphi=\pi / 2)$ and allow the system to relax to steady-state condition.

We now turn our attention towards the creep regime, which is of most importance for field-driven domain-wall motion in ultrathin disordered multilayers [23]. In this case, the creep law predicts [34]

$$
v_{\text {creep }}=v_{0} \exp \left[-\left(T_{c} / T\right)\left(H_{c} / H_{z}\right)^{1 / 4}\right],
$$

where $T_{c}$ is the critical temperature (close to the depinning temperature in fact), $T$ is the sample temperature, $H_{z}$ is the applied field, and $H_{c}$ is the critical field needed to overcome the disorder pinning potential. This expression assumes $H_{z} \ll H_{c}$, and $T_{c} \gg T$. The exponent gathers only terms accounting for the disordered energy landscape of the system and does not include any dissipative contributions in principle. In contrast, the coefficient $v_{0}$ depends on both the energy landscape as well as on the viscosity of the elastic wall. In the limit of an overdamped membrane $\left(\alpha \partial_{t} \mathbf{m} \gg \partial_{t} \mathbf{m}\right)$, $v_{0} \approx \gamma \lambda_{\mathrm{dw}} H_{c} / \alpha$ [34]. It is mostly probable that even for strongly disordered ferromagnets, the creeping domain wall is not in the overdamped regime, although very large damping (up to $\alpha \approx 0.5$ ) has been reported in $\mathrm{Pt} / \mathrm{Co} / \mathrm{AlOx}$ and $\mathrm{Pt} / \mathrm{Co} / \mathrm{Pt}$ systems [3]. However, since the theoretical description of the creep motion of intermediate damped systems is not fully understood, we propose to investigate the impact of chiral damping in this limit.

To evaluate the impact of the chiral damping, we follow the procedure proposed by Je et al. [35] and rewrite the creep law,

$$
v_{\text {creep }}(\mathbf{H})=\frac{\eta_{0} \sigma_{\mathrm{dw}}\left(H_{x}\right)^{\beta}}{\alpha_{\mathrm{eff}}\left(H_{x}\right)} \exp \left(-\chi_{0} \frac{\sigma_{\mathrm{dw}}^{1 / 4}\left(H_{x}\right)}{\sigma_{\mathrm{dw}}^{1 / 4}(0)} H_{z}^{-\frac{1}{4}}\right) .
$$

Here, $\sigma_{\mathrm{dw}}$ is the (chiral) energy of the domain wall, $\beta$ is a coefficient that describes the scaling law between the critical force $H_{c}$ and the domain-wall energy, and $\eta_{0}$ and $\chi_{0}$ are

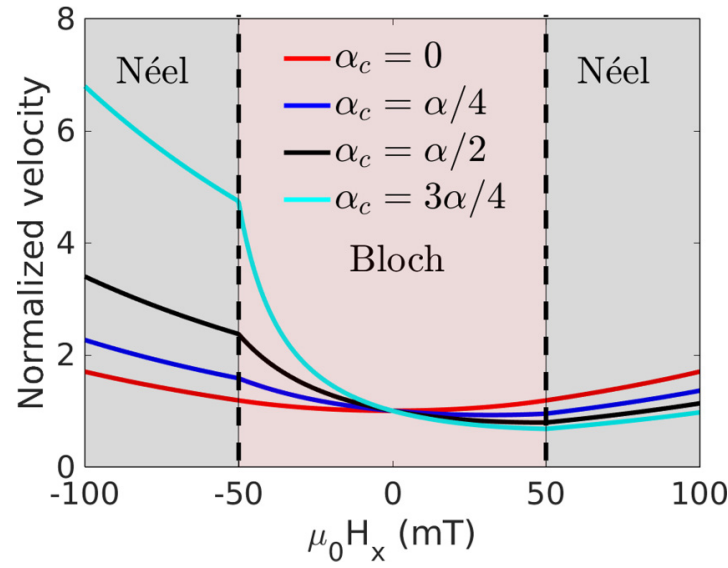

FIG. 3. Normalized domain-wall velocity as a function of inplane magnetic field $H_{x}$ in the presence of a driving field $H_{z}=$ $20 \mathrm{mT}$. The in-plane magnetic field pushes the domain from a Bloch to a Néel configuration and hence increases the azimuthal angle $\varphi$, thereby modifying the damping of the wall. A kink in the velocity is observed at $H_{x}=-H_{k}$, when the domain wall saturates in the Néel configuration when the damping becomes independent of the in-plane field $H_{x}$.

normalization factors that can be chosen to fit experimental data $[23,35]$. The domain-wall energy reads

$$
\sigma_{\mathrm{dw}}=\sigma_{0}+\pi \lambda_{\mathrm{dw}} \mu_{0} M_{s}\left[\frac{1}{2} H_{k} \cos \varphi-H_{x}\right] \cos \varphi,
$$

where the first term $\sigma_{0}$ accounts for the $\varphi$-independent contribution to the magnetic energy, the second term is the magnetic anisotropy field $H_{k}$ favoring Bloch configuration, and the last term $H_{x}$ is the longitudinal field favoring Néel configuration. The energy minimization $\partial_{\varphi} \sigma_{\mathrm{dw}}=0$ gives [35]

$$
\frac{\sigma_{\mathrm{dw}}}{\sigma_{0}}= \begin{cases}1-\left(H_{x}\right)^{2} / H_{\mathrm{dw}} H_{k}: & \left|H_{x}\right| \leqslant H_{k} \\ 1+\left(H_{k}-2\left|H_{x}\right|\right) / H_{\mathrm{dw}}: & \left|H_{x}\right|>H_{k},\end{cases}
$$

where $H_{\mathrm{dw}}=\frac{\pi}{2} \lambda_{\mathrm{dw}} \mu_{0} M_{s} / \sigma_{0}$. The magnetic damping $\alpha_{\mathrm{eff}}$ is written in the simplest form $\alpha_{\text {eff }}=\alpha+s \alpha_{c} \cos \varphi$. To investigate the impact of chiral damping on the creep motion, we chose $H_{\mathrm{dw}}=1 \mathrm{~T}$, and $H_{k}=50 \mathrm{mT}$. The normalized velocity $v\left(H_{x}\right) / v(0)$ of the domain wall as a function of the in-plane field and for different strengths of chiral damping $\alpha_{c}$ is represented in Fig. 3. For $\left|H_{x}\right|<H_{k}$, the velocity varies smoothly while the domain wall changes from one Néel chirality to another. For $\left|H_{x}\right|>H_{k}$, the domain wall saturates in the Néel configuration and the velocity follows the exponential law given above. These results agree with Ref. [23]. Including DMI in the calculation (i.e., $H_{x} \rightarrow H_{x}+s H_{\mathrm{DMI}}$ ) only results in a horizontal shift of the velocity curve in Fig. 3.

\section{CONCLUSION}

The symmetry principles discussed in this work are general and ensure the existence of such a chiral damping in any magnetic structure (ferromagnets, antiferromagnets, chiral magnets, but also metals and insulators etc.) presenting spatial inversion symmetry breaking. The physical mechanisms responsible for this chiral damping can be spin-orbit coupling but also dipolar coupling or magnetic frustrations, as in the 
case of DMI. However, because different orbital characters are involved, chiral damping and DMI do not necessarily scale with each other. The recent observation of a chiral signature in the viscosity of creeping magnetic domain walls [23a] as well as asymmetric damping of spin waves [16] are clear evidence of such a chiral damping in noncentrosymmetric magnetic systems. Finally, chiral damping is not limited to magnetic systems and is expected to have significant impact on the transport properties of systems whose dynamics is dominated by viscosity, such as vortex motion in type-II superconductors, crystal growth, or percolation in porous media [36].

\section{ACKNOWLEDGMENT}

This publication is based upon work supported by the King Abdullah University of Science and Technology (KAUST) through Award No. OSR-CRG URF/1/1693-01 from the Office of Sponsored Research (OSR).
[1] A. V. Kimel, A. Kirilyuk, A. Tsvetkov, R. V. Pisarev, and Th. Rasing, Nature (London) 429, 850 (2004).

[2] C-H. Lambert et al., Science 345, 1337 (2014).

[3] I. M. Miron, G. Gaudin et al., Nat. Mater. 9, 230 (2010); I. M. Miron, T. Moore et al., ibid. 10, 419 (2011).

[4] S. Emori, U. Bauer, S.-M. Ahn, E. Martinez, and G. S. D. Beach, Nat. Mater. 12, 611 (2013).

[5] S. H. Yang, K.-S. Ryu, and S. S. P. Parkin, Nat. Nano. 10, 221 (2015); K.-S. Ryu, L. Thomas, S.-H. Yang, and S. Parkin, ibid. 8, 527 (2013); K.-S. Ryu, S.-H. Yang, L. Thomas, and S. S. P. Parkin, Nat. Commun. 5, 3910 (2014).

[6] I. Dzyaloshinsky, J. Phys. Chem. Solids 4, 241 (1958).

[7] T. Moriya, Phys. Rev. 120, 91 (1960).

[8] N. Nagaosa and Y. Tokura, Nat. Nanotechnol. 8, 899 (2013).

[9] M. Bode et al., Nature (London) 447, 190 (2007); P. Ferriani, K. von Bergmann, E. Y. Vedmedenko, S. Heinze, M. Bode, M. Heide, G. Bihlmayer, S. Blügel, and R. Wiesendanger, Phys. Rev. Lett. 101, 027201 (2008); S. Heinze et al., Nat. Phys. 7, 713 (2011)

[10] W. Jiang et al., Science 349, 283 (2015); S. Woo et al., Nat. Mater. 15, 501 (2016); C. Moreau-Eluchaire et al., Nat. Nanotechnol 11, 444 (2016); O. Boulle et al., ibid. 11, 449 (2016).

[11] M. Heide, G. Bihlmayer, and S. Blügel, Phys. Rev. B 78, 140403(R) (2008).

[12] G. Chen, J. Zhu et al., Phys. Rev. Lett. 110, 177204 (2013); G. Chen, T. Ma et al., Nat. Commun. 4, 2671 (2013).

[13] J.-P. Tetienne et al., Nat. Commun. 6, 6733 (2015).

[14] A. Thiaville, S. Rohart, E. Jué, V. Cros, and A. Fert, Europhys. Lett. 100, 57002 (2012).

[15] K. Zakeri, Y. Zhang, J. Prokop, T. H. Chuang, N. Sakr, W. X. Tang, and J. Kirschner, Phys. Rev. Lett. 104, 137203 (2010).

[16] Kh. Zakeri, Y. Zhang, T.-H. Chuang, and J. Kirschner, Phys. Rev. Lett. 108, 197205 (2012).

[17] J.-H. Moon, S. M. Seo, K. J. Lee, K. W. Kim, J. Ryu, H. W. Lee, R. D. McMichael, and M. D. Stiles, Phys. Rev. B 88, 184404 (2013); A. Manchon, P. B. Ndiaye, J. H. Moon, H. W. Lee, and K. J. Lee, ibid. 90, 224403 (2014).

[18] D. L. Mills and S. M. Rezende, Top. Appl. Phys. 87, 27 (2003).

[19] V. Kambersky, Czech. J. Phys. B 26, 1366 (1976); Phys. Rev. B 76, 134416 (2007); K. Gilmore, Y. U. Idzerda, and M. D. Stiles, J. Appl. Phys. 103, 07D303 (2008).
[20] S. Zhang and Steven S.-L. Zhang, Phys. Rev. Lett. 102, 086601 (2009); J. Foros, A. Brataas, Y. Tserkovnyak, and G. E. W. Bauer, Phys. Rev. B 78, 140402 (2008).

[21] H. T. Nembach, J. M. Shaw, C. T. Boone, and T. J. Silva, Phys. Rev. Lett. 110, 117201 (2013); Y. Li and W. E. Bailey, ibid. 116, 117602 (2016).

[22] Z. Yuan, K. M. D. Hals, Y. Liu, A. A. Starikov, A. Brataas, and P. J. Kelly, Phys. Rev. Lett. 113, 266603 (2014).

[23] (a) E. Jué, C. K. Safeer, M. Drouard, A. Lopez, P. Balint, L. Buda-Prejbeanu, O. Boulle, S. Auffret, A. Schuhl, A. Manchon, I. M. Miron, and G. Gaudin, Nat. Mater. 15, 272 (2016); (b) R. Lavrijsen, D. M. F. Hartmann, A. van den Brink, Y. Yin, B. Barcones, R. A. Duine, M. A. Verheijen, H. J. M. Swagten, B. Koopmans, Phys. Rev. B 91, 104414 (2015).

[24] K. M. D. Hals and A. Brataas, Phys. Rev. B 89, 064426 (2014).

[25] K.-W. Kim, J.-H. Moon, K.-J. Lee, and H.-W. Lee, Phys. Rev. Lett. 108, 217202 (2012); J.-V. Kim, Phys. Rev. B 92, 014418 (2015).

[26] X. Wang, C. O. Pauyac, and A. Manchon, Phys. Rev. B 89, 054405 (2014).

[27] K.-W. Kim, H.-W. Lee, K.-J. Lee, and M. D. Stiles, Phys. Rev. Lett. 111, 216601 (2013).

[28] S. E. Barnes and S. Maekawa, Phys. Rev. Lett. 98, 246601 (2007).

[29] Y. Tserkovnyak, A. Brataas, and G. E. W. Bauer, Phys. Rev. Lett. 88, 117601 (2002).

[30] See Supplemental Material at http://link.aps.org/supplemental/ 10.1103/PhysRevB.93.214429 for the derivation of Eqs. (5) and (8).

[31] V. Kashid, T. Schena, B. Zimmermann, Y. Mokrousov, S. Blügel, V. Shah, and H. G. Salunke, Phys. Rev. B 90, 054412 (2014).

[32] G. Tatara, N. Nakabayashi, and K. J. Lee, Phys. Rev. B 87, 054403 (2013); G. Tatara et al., J. Appl. Phys. 115, 172609 (2014).

[33] S. Zhang and Z. Li, Phys. Rev. Lett. 93, 127204 (2004).

[34] P. Chauve, T. Giamarchi, and P. Le Doussal, Phys. Rev. B 62 , 6241 (2000).

[35] S.-G. Je, D. H. Kim, S. C. Yoo, B. C. Min, K. J. Lee, and S. B. Choe, Phys. Rev. B 88, 214401 (2013).

[36] Y. Bugoslavsky, G. K. Perkins, X. Qi, L. F. Cohen, and A. D. Caplin, Nature (London) 410, 563 (2001); D. Wilkinson and J. F. Willemsen, J. Phys. A 16, 3365 (1983); Y. Chen and C. A. Schuh, Phys. Rev. Lett. 98, 035701 (2007). 